\section{CHANGING VERBAL LABEL ASSIGNMENTS SELECTS THE MEMORY SYSTEM FOR RESPONSES IN AN IMMEDIATE VISUAL RECOGNITION TASK}

\author{
ALEXA BECKER, MENGXUE KANG, \\ ARNOLD GLASS (FACULTYADVISOR)
}

\section{次}

\section{ABSTRACT}

The dual system hypothesis posits the existence of two neural systems for memory and learning in the mammalian brain: the habit system and the improvisational system. This study sought to determine whether both systems are involved in a visual recognition task originally outlined in Sternberg (1966) and whether each system could be selectively engaged on the basis of response assignment. ${ }^{[13]}$ Seventeen undergraduate students participated in an immediate visual recognition task where they responded whether or not a test consonant was present in a previous study sequence of one to six consonants by pressing one key for same or another key for different. When the different response was assigned to the spatially right " $\mathrm{J}$ " key, reaction time for targets and lures was a function of the study sequence size, indicating that the study sequence was serially scanned and compared with the test item by the habit system. However, when the same response was assigned to the spatially right " $\mathrm{J}$ " key, reaction time was not a function of study sequence size, indicating that the test item was not compared with the study sequence and responses were instead determined by perceived recency/novelty of the test item by the improvisational system. Differences in reaction time depending on response assignment suggest the selection of one memory system over the other based on verbal labels assigned to response keys in different spatial locations. Verbal label refers to the label of same or different assigned to the response keys in the experiment instructions. Results expand upon Sternberg (1966)-which used the same visual recognition task design as this study but did not account for response assignment, obscuring evidence of contributions from both memory systems-and provide more evidence for the dual-system hypothesis by demonstrating the involvement of both memory systems in immediate visual recognition. ${ }^{[13]}$

\section{$1 \quad$ INTRODUCTION}

Sternberg's (1966) seminal study on memory scanning found that, for a study sequence of one to six digits presented one at a time, visual recognition response time (RT) for a test digit was an increasing function of the length of the study sequence for both targets (a test digit that was present in the study sequence) and lures (a test digit that was not present in the study sequence). ${ }^{[13]}$ These results suggested the existence of a visual recognition system that computes by (exhaustively) serially scanning a representation of a study set for a match with a test item. Less than a decade after Sternberg's study, evidence was found for the existence of an additional visual recognition system that computes a test item's perceived recency (referring to a test item that one recognizes as just having been seen in a previous study set) or novelty (referring to a new test item that one does not recognize as just having been seen in a previous study set) without scanning through a representation of a corresponding study set. Whereas Sternberg's results suggested the serial scanning of a study set in memory for a match with a test item, Atkinson \& Juola's (1973 \& 1974) suggested a purely perceptual judgment of the recency/novelty of a test item without comparison to a study set. ${ }^{[1,2]}$ Sternberg and Atkinson \& Juola both 
found evidence for two different systems of visual recognition; however, researchers at the time had no explanation for why there would be more than one.

Evidence of the contributions of two distinct neural systems to animal navigation provided the first evidence of two distinct neural systems for recognition. This led to the proposal of the dual-system hypothesis, a possible resolution to the mystery of Sternberg and Atkinson \& Juola's findings. ${ }^{[9,11]}$ The dual-system hypothesis posits that two integrated, yet distinct systems of learning and memory exist in the mammalian brain: the improvisational system and the habit system. The improvisational system includes the hippocampus and surrounding medial temporal regions, the inferior temporal cortex, and the occipital cortex. It is responsible for constructing visual representations of the world and making responses to novel targets. The habit system includes the basal ganglia, the supplementary, premotor, and ventrolateral areas of the frontal cortex, the parietal cortex, and occipital cortex. It is responsible for encoding and serially generating sequences of actions. ${ }^{[5]}$ See FIGURE 1 for an fMRI scan depicting portions of these systems. A variety of behavioral findings-such as spatial navigation, habit formation, and goal-directed actions-have been found to have a clear neural basis in the context of this dual-system model. ${ }^{[15]}$

The improvisational system makes recognition judgments based on a test item's perceived recency or novelty. ${ }^{[14]}$ The most likely explanation for how the improvisational system computes recency/novelty is on the basis of the neural response to a perceptual stimulus. A repeated visual target activates exactly the same neurons, and the response of those neurons decreases as a function of the repetition due to habituation. So, if a test item was just shown in a previous study set, the improvisational system will detect that habituated (hence reduced) neural pattern and compute recency (a match with one of the just-seen study items). If it does not detect a habituated neural pattern upon presentation of the test item, it will compute novelty (a new item; no match). Well before the establishment of the dualsystem hypothesis, Groves and Thompson (1970) first demonstrated that this was a plausible basis for the detection of recency/novelty. ${ }^{[6]}$ Contrarily, the habit system makes recognition judgments by retrieving a representation of the study items from memory and serially scanning the set of study items
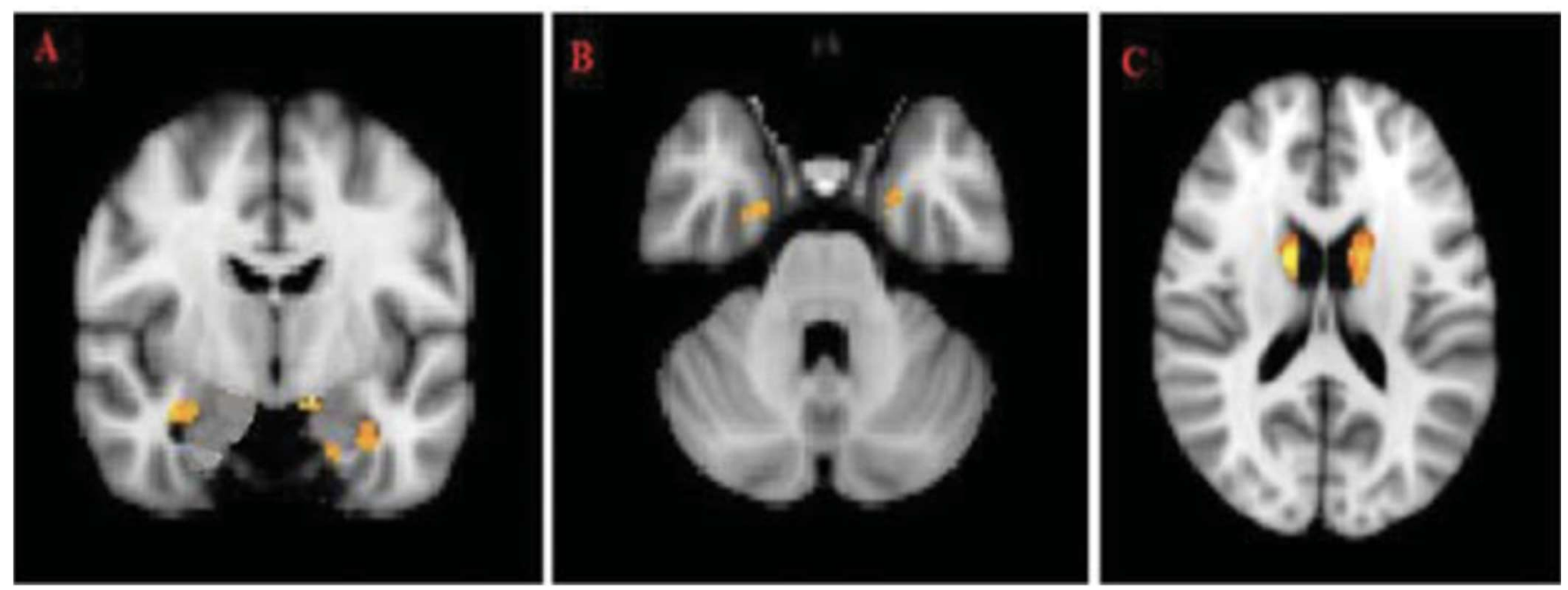

FIGURE 1: Clusters in the left/right hippocampus (A) and left/right anterior parahippocampal gyrus (B), which are part of the improvisational system. (C) shows clusters in the left/right caudate, which is part of the habit system. A better illustration of the habit and improvisational systems does not yet exist unfortunately, but these scans from Sinha and Glass (2017) show the crucial parts of each system. 
for a match with a test item. ${ }^{[3]}$

Sinha \& Glass (2017) found evidence for the involvement of both of these memory systems in a same/different matching task where participants judged whether two four-consonant strings were the same or different (e.g., study string "BDCF" vs. test string "BCFD"). ${ }^{[12]}$ Participants were presented with a study string followed by a test string to which they had to respond with either same or different. RT for different responses was an increasing function of the left-to-right location of the first difference between study and test strings, indicating that the habit system made different responses by serially comparing the consonants in the study and test strings from left to right. It follows from this line of reasoning that same responses ought to be the slowest since the participant presumably must compare all four test string items to all four study string items to determine that they are the same. However, RT for same responses was faster than different responses, indicating that the improvisational system was making same responses based on the perceived recency of the test string as a whole chunk without serially comparing each item in the study and test strings. fMRI results corroborated this interpretation and showed that different responses were associated with activity in both the caudate and hippocampus while same responses were associated with just hippocampal activity.

Based on a connectivity analysis of this brain activity, Sinha \& Glass (2017) posited that when the test string is presented, the improvisational system begins a holistic perceptual comparison between the study and test strings while the habit system simultaneously initiates a serial left-to-right comparison of the individual study and test string consonants. ${ }^{[12]}$ During this holistic comparison, mental maps of the test and study strings are compared; if the strings match across all four positions, a strong match signal is produced by the hippocampus and a same response is made. When the study and test strings are identical, the match signal produced by the hippocampus is powerful enough to inhibit the serial comparison process initiated by the caudate and produce a same response. When the study and test strings differ, the match signal is not strong enough to inhibit the serial comparison process, so the habit system takes over and the serial comparison continues until a mismatch is found and a different response is then made.

The functional roles of the improvisational and habit systems in a visual recognition task where the study and test items were both four-consonant strings were evident from the results of Sinha \& Glass (2017). ${ }^{[12]}$ The two systems appear to work in parallel and one of them responds depending on whether the test string is the same as or different from the study string. However, there was still the question of how the systems would function when the test item was a single consonant. Kang, Norman, Zhou, Becker, \& Glass (2021) replicated the same experimental design as Sinha \& Glass (2017) with a fourconsonant study string, except the test item was a single consonant that either was (eliciting a same response) or was not (eliciting a different response) present in the study string. They found that when the different response was assigned to the spatially right response key and the same response was assigned to the spatially left response key, RT for same responses to targets was an increasing function of the test consonant's position in the study string, indicating that the habit system made responses by serially scanning the study string until a match (if there was one) with the test consonant was found. However, when the response assignment was switched-the different response was assigned to the spatially left response key and the same response was assigned to the spatially right response key-RT for same responses was not significantly different among the four possible positions in the study string. This indicated that the improvisational system made responses based on the perceived recency/novelty of the test consonant without scanning the study string for a match. ${ }^{[7]}$ Kang et al. (2021) also conducted an iteration of this experiment where participants used two fingers on the same hand to make responses with keys in different spatial locations. They found the same result, confirming that the assignment of a verbal label to a key in a relative right or left spatial position determines which memory system is selected for the task. This refutes an alternative explanation that the assignment of verbal label same or 
different to a specific hand causes the hemisphere associated with that hand to control which memory system is selected for the task. ${ }^{[7]}$ Therefore, when the study item is a four-consonant string and the test item is a single consonant, one system is selected to make responses in the task over the other based on the assignment of verbal labels to response keys in different spatial locations.

Sternberg (1966) did not take into account the effect of response assignment on RT; since it is not mentioned anywhere in his paper, he either only tested one response assignment or varied the response assignment but did not discriminate between RTs from trials of different response assignments in his analyses. The purpose of this experiment was to investigate the effect of response assignment on RT by designating it as a separate independent variable in a replication of Sternberg (1966). The researchers aim to determine whether response assignment influences the control of the improvisational versus habit system in Sternberg's immediate visual recognition task. Based on the results of Kang et al. (2021) where both memory systems were differentially involved in the task depending on the response assignment, the researchers hypothesize that the finding of contributions from both memory systems in Sternberg (1966) may have been obscured originally by his disregard for response assignment's effect on RT. Thus, it could be the case that both memory systems are indeed involved in this task and that one system is selected to make responses over the other based on response assignment.

\section{Methodology}

Participants were seventeen students distributed across two psychology courses at Rutgers University-New Brunswick in New Jersey. At the time that this study was run, the researchers did not have access to a larger online subject pool; however, one researcher had remote access to a pool of students enrolled in his psychology courses and the study here was relevant to their course material, so they were used as subjects. Participants consented to taking part in experiments as a feature of the course they were enrolled in and received course credit for their participation. Eleven were female and six were male, aged 18-23. Sixteen were right-handed and one was left-handed based on self-reports of handedness.

Stimuli were capitalized consonants randomly selected from a pool of all consonants (excluding Y). In a previous replication of Sternberg (1966), Glass (1993) used three different kinds of stimuli in Sternberg's original design: digits, consonants from the first half of the alphabet, and consonants from the second half of the alphabet. ${ }^{[4]}$ There was no difference in results based on the stimuli. Study sequences were one to six consonants in length. Each consonant was presented one at a time for 1.2 seconds each with no inter-stimulus intervals, consistent with the design and timing in Sternberg (1966). In the "Right Key = Different" response assignment condition, participants responded by pressing the spatially left " $F$ " key if the test consonant was present in the study sequence (a same response) and the spatially right " $\mathrm{J}$ " key if it was not (a different response). In the "Right Key = Same" condition, participants had the opposite response assignment. Each participant completed one session with the "Right Key = Different" response assignment and one session with the "Right Key = Same" response assignment. Participants were randomly assigned to one of two groups: group one $(\mathrm{N}=9)$ completed the "Right Key = Different" session first and the "Right Key $=$ Same" session second. Group two $(N=8)$ completed the sessions in the opposite order..$^{[a]}$

All participants completed twenty practice trials at the beginning of each session to understand how the trials would run. RTs from practice trials were not included in the larger analysis. There were an equal number of regular trials for each possible

\footnotetext{
[a] The participants in this experiment were students from two different Rutgers courses. "Course A" completed the experiment first, running 144 regular trials per session. After observing significant results from "course $A$ ", the researchers increased the regular trials for "course $B$ " to 360 per session to see if the effect observed in the results of "course $A$ " remained. Of the sample of 17, 10 participants were in "course $A$ " and 7 participants were in "course B". Groups 1 and 2 mentioned above-which are the groups the researchers are comparing-are each composed of students from both of these courses. The final results detailed later are a combination of the results of both courses, but these combined results do not significantly differ from the initial results of just "course A" (data not shown).
} 
study sequence size (one to six). Within the group of trials for a particular study sequence size, half of the subsequent test consonants were targets (test items presented in the study sequence) and half were lures (test items not presented in the study sequence). Of the trials where the test consonant was a target, there were an equal number of trials for each possible position of the test consonant in the study sequence. The experiment was administered remotely; participants were given Python code files containing the experiment code and instructions on how to run it through PsychoPy on their personal computers. ${ }^{[10]}$

Before the trials began, instructions were presented (see FIGURES 2A \& 2B). Though there were no explicit instructions regarding which hands or fingers to use to respond, the researchers assume participants used their left hand to press the " $F$ " key and their right hand to press the " $\mathrm{J}$ " key as that is the normal typing position. However, the researchers are more concerned with the spatial location of the response keys rather than which hands the participants used to respond; as explained earlier, it is the assignment of verbal labels to response keys in different spatial locations that determines the memory system (and thus visual recognition method) used to respond, not the assignment of verbal labels to different hands. ${ }^{[7]}$

For each trial, a study sequence of one to six capitalized consonants was shown-one consonant at a time in the center of the screen-and then a fixation asterisk was presented followed by a test consonant. The purpose of the fixation asterisk was to focus the participant's gaze to a particular point (the center of the screen) where the test consonant was about to be displayed so that RT did not include the time it takes for a participant to direct their gaze towards the test consonant, only the time it took for them to respond. The fixation asterisk was black for two seconds and then red for one second to indicate that the test consonant was about to appear. Participants responded as fast as possible whether the test consonant had been present in the previous sequence. The test consonant disappeared immediately after a response was made and feedback on accuracy was presented, as was done in Sternberg

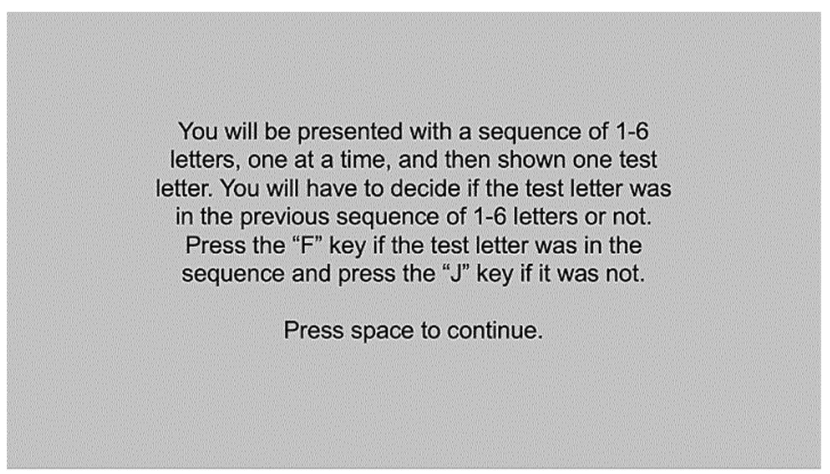

FIGURE 2A: First instructions screen displayed upon opening the experiment.

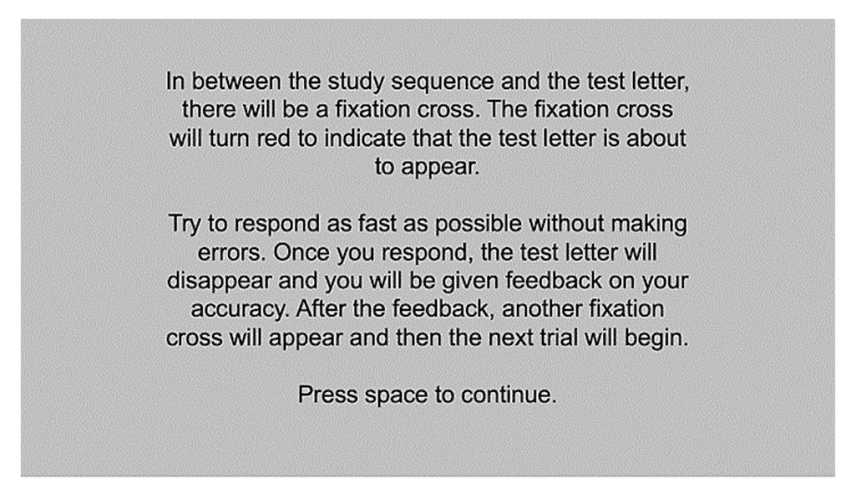

FIGURE 2B: Second instructions screen.

(1966). ${ }^{[13]}$ Feedback was one of four possible sentences (depending on the response) displayed for three seconds that said "Correct, the test letter was/was not in the sequence" or "Incorrect, the test letter was/was not in the sequence". Fixation asterisks appeared in between successive trials as well.

\section{RESULTS}

A within-subjects ANOVA was selected to assess the effect of response assignment condition ("Right Key = Different" or "Right Key = Same"), judgment (same response or different response), and study sequence size (1-6 consonants) on RT. The researchers selected a within-subjects ANOVA because each subject completed one session for each response assignment, and they wanted to measure the difference in RT for those conditions for each subject. All variables were within-subjects variables. 
The degrees of freedom, F-statistic, p-value, and effect size for all variables and interactions are contained in TABLE 1.

The effects of response assignment and judgment were not significant. The effect of study sequence size was significant, $p=0.002$. The interaction of response assignment and judgment was significant, $p=0.034$. The interaction of response assignment and study sequence size was significant, $p=0.013$. There was an effect of study sequence size on RT in the "Right Key = Different" condition for targets $(p<0.001)$ and lures $(p<0.001)$, but there was no effect of study sequence size on RT in the "Right Key = Same" conditions for targets or lures. This result is shown in FIGURE 3, which includes the average RT for each condition/study sequence size across all subjects. None of the other interactionsbetween judgment and study sequence size and between condition, judgment, and study sequence size-were significant.

\begin{tabular}{|c|c|c|c|c|}
\hline VARIABLE/INTERACTION & $\begin{array}{l}\text { DEGREES OF } \\
\text { FREEDOM }\end{array}$ & F-STATISTIC & P-VALUE & $\begin{array}{c}\text { EFFECT SIZE } \\
\text { (OBSERVED POWER) }\end{array}$ \\
\hline RESPONSE ASSIGNMENT (RA) & 1 & 0.647 & 0.433 & 0.118 \\
\hline JUDGMENT & 1 & 2.605 & 0.126 & 0.329 \\
\hline STUdy SEQUENCE SIZE & 5 & 4.285 & $0.002^{* *}$ & 0.952 \\
\hline RA $X$ JUDGMENT & 1 & 5.353 & $0.034^{*}$ & 0.585 \\
\hline RA $X$ STUdy SEQUENCE SIZE & 5 & 3.086 & $0.013^{*}$ & 0.850 \\
\hline $\begin{array}{l}\text { RA } x \text { STUDY SEQUENCE SIZE } \\
\text { (RIGHT KEY }=\text { DIFFERENT- TARGETS) }\end{array}$ & 5 & 7.383 & $<0.001^{* *}$ & 0.999 \\
\hline $\begin{array}{l}\text { RA } X \text { STUDY SEQUENCE SIZE } \\
(\text { RIGHT KEY = DIFFERENT - LURES) }\end{array}$ & 5 & 14.189 & $<0.001^{* *}$ & 1.000 \\
\hline $\begin{array}{l}\text { RA } X \text { STUDY SEQUENCE SIZE } \\
(\text { RIGHT KEY }=S A M E-\text { TARGETS) }\end{array}$ & 5 & 0.419 & 0.834 & 0.154 \\
\hline $\begin{array}{l}\text { RA } X \text { STUDY SEQUENCE SIZE } \\
(\text { RIGHT KEY }=S A M E-\text { LURES) }\end{array}$ & 5 & 0.962 & 0.446 & 0.327 \\
\hline JUDGMENT $X$ STUDY SEQUENCE SIZE & 5 & 0.852 & 0.536 & 0.281 \\
\hline RA $X$ JUdGMENT $X$ STUdy SEQUENCE SizE & 5 & 0.402 & 0.846 & 0.150 \\
\hline
\end{tabular}

TABLE 1: $*$ indicates $p<0.05 . * *$ indicates $p<0.01$. 


\section{Study Sequence Size vs. Reaction Time for Targets and Lures in Each Condition}

0.9

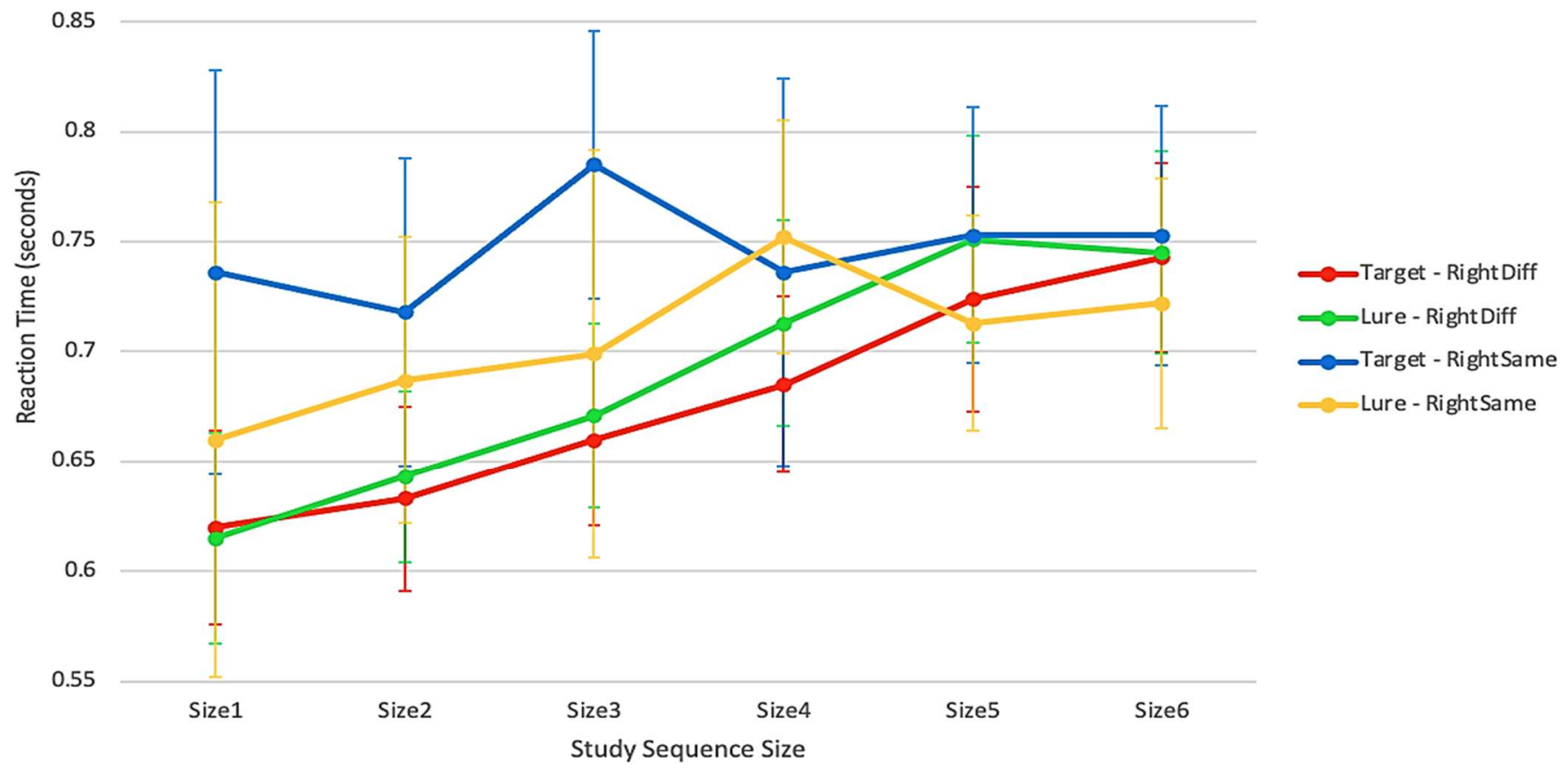

FIGURE 2: RT is only a function of study sequence size in the "Right Key = Different" condition, statistically verified by the withinsubjects ANOVA. RTs (y-axis) are averages for the entire set of participants. Error bars represent the standard error of the sampling distribution of each condition/study set size, specified in the table below (TABLE 2).

\begin{tabular}{|c|c|c|c|c|}
\cline { 2 - 5 } \multicolumn{1}{c|}{} & $\begin{array}{c}\text { TARGET - RIGHT KEY } \\
=\text { DIFFERENT }\end{array}$ & $\begin{array}{c}\text { LURE - RIGHT KEY } \\
=\text { DIFFERENT }\end{array}$ & $\begin{array}{c}\text { TARGET - RIGHT KEY } \\
=\text { SAME }\end{array}$ & $\begin{array}{c}\text { LURE - RIGHT KEY } \\
=\text { SAME }\end{array}$ \\
\hline SIZE 1 & 0.044 & 0.048 & 0.108 & 0.092 \\
\hline SIZE 2 & 0.042 & 0.039 & 0.065 & 0.07 \\
\hline SIZE 3 & 0.039 & 0.042 & 0.093 & 0.061 \\
\hline SIZE 4 & 0.04 & 0.047 & 0.053 & 0.088 \\
\hline SIZE 5 & 0.051 & 0.047 & 0.049 & 0.058 \\
\hline SIZE 6 & 0.043 & 0.046 & 0.057 & 0.059 \\
\hline
\end{tabular}

TABLE 2: Values for the standard error bars in FIGURE 3. 


\section{4}

\section{DISCUSSION}

The purpose of this experiment was to investigate the effect of response assignment on RT in a replica of Sternberg (1966). The researchers hypothesized that Sternberg's disregard for response assignment's effect on RT may have obscured evidence for contributions from two memory systemshence two methods of visual recognition-in his task. The finding of significant differences in RT between the "Right Key = Different" and "Right Key = Same" conditions suggested that serial scanning of the study sequence by the habit system only occurred in the "Right Key = Different" condition. It was only in this condition that RT was a function of study sequence size, indicating serial comparison of the test item to each item in the study sequence; longer study sequences require more time to serially scan, and thus result in longer RTs. The fact that RT was not a function of study sequence size in the "Right Key= Same" condition suggests that the improvisational system made judgments of the perceived recency/novelty of the test consonant (based on detection of a habituated/non-habituated neural pattern, respectively) without comparing it to the study sequence. This expands upon Sternberg's original findings and provides more evidence for the dualsystem hypothesis. Our results suggest that both the habit system and the improvisational system are indeed involved in this task, and an experimenter can control the memory system selected to make responses in the task by assigning different verbal labels to response keys in different spatial locations (in this case, different keys on a keyboard).

The researchers do not have a definite answer as to why the habit system is selected for the task when the different verbal label is assigned to the spatially right response key and the same verbal label is assigned to the spatially left response key (Right Key = Different condition) while the improvisational system is selected for the task when the response assignment is the opposite (Right Key = Same condition). Even though Kang et al. (2021) confirmed that the assignment of verbal labels to response keys in different spatial locations determines which memory system gets selected for the task-not the assignment of verbal labels to different hands- there is still no clear answer as to why the two response assignment conditions result in the selection of different memory systems. For the same task, why should merely switching the verbal labels assigned to the response keys cause the participant to use a different memory system? The researchers suggest that the verbal label assigned to the spatially right key could be the deciding factor. Language is localized in the left hemisphere, which processes information from the right side of space. Therefore-since the verbal label assigned to the spatially right response key is initially processed by the languagedominant left hemisphere while the verbal label assigned to the spatially left response key is initially processed by the non-language-dominant right hemisphere-the language-dominant left hemisphere may interpret the task and select the appropriate memory system depending on verbal label assigned to the spatially right response key. Perhaps when the label of different is assigned to the spatially right response key (Right Key = Different condition), the experiment is interpreted as a difference-detection task that implies serial comparison, and so the habit system is selected to make responses. Conversely, when the label of same is assigned to the spatially right response key (Right Key = Same condition), the experiment is interpreted as a recency/novelty detection task that implies a purely perceptual judgement, and so the improvisational system is selected to make responses. However, this is merely a suggestion; the verbal label assigned to the spatially left key could instead be the determining factor, or it could be the spatial position of both keys.

To determine if it is indeed the verbal label processed by the language-dominant hemisphere that controls which system is selected for the task, future studies ought to measure participant language dominance directly by using brain imaging techniques to see if the location of one's language center has any effect on their responses. If individuals with atypical right hemisphere language localization use the opposite neural system as their typical counterparts to respond when the response assignment is the same, this would provide evidence for the notion that it is the verbal label processed by the language- 
dominant hemisphere that controls the memory system selected for the task. There is an increased incidence of atypical right hemisphere language localization in strongly left-handed individuals. ${ }^{[8]}$ However, it only occurs in about $25 \%$ of those individuals, and there was no evidence that the one left-handed participant in this study was using the opposite neural system as the other participants to respond when the response assignment was the same. They were also not an outlier regarding RT.

A limitation of our experiment is that due to the move to remote instruction as a consequence of the COVID-19 pandemic, participants completed this experiment in their own homes at their leisure, so environmental confounds could not be controlled as they normally would be in a laboratory setting. However, this experiment could still better control for those confounds while remaining remote, perhaps by running the sessions over video call to monitor participants. The sample size was also small, and the sizes of each group were slightly uneven (group $1, N=9$; group 2, $N=8$ ). Participant fatigue could have also been a confounding factor as breaks did not appear during any of the sessions. Further replications of this experiment that include fMRI recordings ought to be done to see if activation in the caudate/hippocampus like that observed in Sinha \& Glass (2017) and Kang et al. (2021) is found during this task

\section{5}

\section{ACKNOWLEDGEMENTS}

This research was conducted under Dr. Arnold Glass in his lab. Data analyses referenced in the results section were facilitated by our lab's graduate student, Mengxue Kang.

\section{REFERENCES}

[1] Atkinson, R. C., \& Juola, J. F. (1973). Factors influencing speed and accuracy of word recognition. Fourth international symposium on attention and performance, (583-611).

[2] Atkinson, R. C., \& Juola, J. F. (1974). Search and decision processes in recognition memory. Contemporary developments in mathematical psychology: Vol. 1. Learning, memory \& thinking.

[3] Checkosky, S. F., \& Baboorian, N. (1972). Memory search for CVC and CCC trigrams. Journal of Experimental Psychology, 96(1), 158-163.

[4] Glass, A.L. (1993). The role of generation in recognition. Scandinavian Journal of Psychology, 34(3), 255-267

[5] Glass, A. L. (2019). Within the framework of the dual-system model, voluntary action is central to cognition. Attention, Perception, \& Psychophysics, 81(7), 2192 - 2216.

[6] Groves, P.M., \& Thompson, R.F. (1970). Habituation: A dualprocess theory. Psychological Review, 77(5), 419-450.

[7] Kang, M., Norman, M., Zhou, W., Becker, A., \& Glass, A. L. (2021). Spatial location of response key selects the neural system for visual recognition (submitted, under review)

[8] Knecht, S., Dräger, B., Deppe, M., Bobe, L., Lohmann, H., Flöel, A., Ringelstein, E. B., Henningsen, H. (2000). Handedness and hemispheric language dominance in healthy humans. Brain, 123(12), 2512-2518

[9] Packard, M. G., \& McGaugh, J. L. (1996). Inactivation of hippocampus or caudate nucleus with lidocaine differentially affects expression of place and response learning. Neuropsychology of Learning and Memory, 65, 65-72.

[10] Peirce, J. W. (2007). PsychoPy - Psychophysics software in Python. Journal of Neuroscience Methods, 162, 8-13.

[11] Poldrack, R. A., \& Packard, M. G. (2003). Competition among multiple memory systems: Converging evidence from animal and human studies. Neuropsychologia, 41, 245-251.

[12] Sinha, N., \& Glass, A. L. (2017). Dissociating Medial Temporal and Striatal Memory Systems With a Same/Different Matching Task: Evidence for Two Neural Systems in Human Recognition. The Journal of General Psychology.

[13] Sternberg, S. (1966). High-speed scanning in human memory. Science, 153(3736), 652-654.

[14] Suzuki, W. A., \& Naya, Y. (2014). The perirhinal cortex. Annual Review of Neuroscience, 37, 39-53.

[15] Yin, H. H., \& Knowlton, B. J. (2006). The role of the basal ganglia in habit formation. Nature Reviews Neuroscience, 7, 464476.

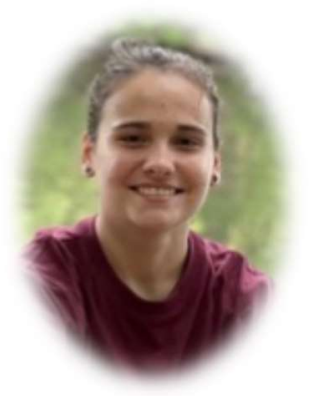

My name is Alexa Becker. I am a rising senior majoring in Psychology and Cognitive Science at Rutgers-New Brunswick. My hobbies include playing the bass, reading, and powerlifting. My research interests include visual recognition and, more broadly, the neural systems involved in memory and learning as well as aging and memory. I have been working in Dr. Arnold Glass' research lab for two years. Dr. Glass has been a professor of psychology at Rutgers for 46 years.

The experiment outlined in this paper was done virtually (as a result of the COVID-19 pandemic). I composed the write-up, designed this experiment, provided participants with materials needed to complete the experiment remotely, and collected results data. Our lab's former graduate student, Mengxue Kang, assisted with designing the experiment and facilitated the statistical analyses. She has since successfully defended her dissertation and now works as a data scientist for CVS 